\title{
Engineered acetoacetate-inducible whole-cell biosensors based on the AtoSC two-component system
}

\author{
Jack W. Rutter ${ }^{1}$ | Linda Dekker ${ }^{1}$ | Alex J. H. Fedorec ${ }^{1}$ | David T. Gonzales ${ }^{1}$ | \\ Ke Yan Wen ${ }^{1}$ | Lewis E. S. Tanner ${ }^{1}$ | Emma Donovan ${ }^{1}$ | Tanel Ozdemir ${ }^{1}$ | \\ Geraint M. Thomas ${ }^{1}$ | Chris P. Barnes ${ }^{1,2}$
}

${ }^{1}$ Department of Cell and Developmental Biology, University College London,

London, UK

${ }^{2}$ Department of Genetics, Evolution and Environment, University College London, London, UK

\section{Correspondence}

Geraint M. Thomas and Chris P. Barnes, Department of Cell and Developmental Biology, University College London, London WC1E 6BT, UK.

Email: g.thomas@ucl.ac.uk and christopher. barnes@ucl.ac.uk

\section{Funding information}

Biotechnology and Biological Sciences Research Council, Grant/Award Number: BB/S003681/1; H2O2O European Research Council, Grant/Award Number: 770835; Wellcome Trust, Grant/Award Number: 209409/Z/17/Z

\begin{abstract}
Whole-cell biosensors hold potential in a variety of industrial, medical, and environmental applications. These biosensors can be constructed through the repurposing of bacterial sensing mechanisms, including the common two-component system (TCS). Here we report on the construction of a range of novel biosensors that are sensitive to acetoacetate, a molecule that plays a number of roles in human health and biology. These biosensors are based on the AtoSC TCS. An ordinary differential equation model to describe the action of the AtoSC TCS was developed and sensitivity analysis of this model used to help inform biosensor design. The final collection of biosensors constructed displayed a range of switching behaviours at physiologically relevant acetoacetate concentrations and can operate in several Escherichia coli host strains. It is envisaged that these biosensor strains will offer an alternative to currently available commercial strip tests and, in future, may be adopted for more complex in vivo or industrial monitoring applications.
\end{abstract}

\section{KEYWORDS}

acetoacetate, biosensors, E. coli, synthetic biology, two-component systems

\section{1 | INTRODUCTION}

Bacterial cells have evolved the ability to detect and respond to environmental cues. The repurposing of these natural abilities to create engineered biosensor strains has become a major area of research within synthetic biology. Whole-cell biosensors, which contain this sensing machinery within a single living cell, offer a number of advantages over more conventional sensing technologies including portability, self-replication, sensing of the bioavailable fraction (rather than total concentration) and low-cost of production (Courbet et al., 2015; Hicks et al., 2020; Jung et al., 2018; Wang et al., 2019). To date, whole-cell biosensors have been developed for the detection of metals in environmental samples (mercury, Mahbub et al., 2017; arsenic, Stocker et al., 2003; lead, Jia et al., 2018), for the monitoring of metabolites during bioproduction (lactate, Goers et al., 2017) and in medical applications for the monitoring of clinically relevant biomarkers (Daeffler et al., 2017; Landry et al., 2018; Lin et al., 2019; Riglar et al., 2017; Woo et al., 2020), exploiting the ability of bacteria to reach inaccessible areas of the host in vivo (Courbet et al., 2015). Whole-cell biosensors can be based on a number of bacterial sensing mechanisms. These include transcription-factor mechanisms, one-component systems, twocomponent systems (TCSs), extracytoplasmic function sigma

This is an open access article under the terms of the Creative Commons Attribution License, which permits use, distribution and reproduction in any medium, provided the original work is properly cited.

(c) 2021 The Authors. Biotechnology and Bioengineering published by Wiley Periodicals LLC 
factors, and other environment responsive mechanisms (Hicks et al., 2020; Wen et al., 2020).

TCSs are one of the most common bacterial sensing mechanisms, with many examples found within bacterial genomes (Hoch, 2000; Stock et al., 2000). Escherichia coli alone are reported to have 32 TCSs (Stock et al., 2000). TCSs typically consist of a membrane-bound histidine kinase $(\mathrm{HK})$ that detects a specific signal. This signal activates kinase activity and causes autophosphorylation of a conserved histidine residue. The phosphoryl group is then transferred to an aspartate residue on the response regulator (RR) (Hoch, 2000). Usually RRs are transcriptional regulators capable of activating or repressing expression from a specific promoter (Mascher et al., 2006), thereby controlling gene expression in response to an external input. TCSs respond to a wide range of input signals, including quorumsensing molecules (Papenfort \& Bassler, 2016), certain wavelengths of light (Duanmu et al., 2014), physical contact (OConnor et al., 2012), and oxidative stress (Singh, 2000). Previously reported TCS whole-cell biosensors have been developed for tetrathionate (Riglar et al., 2017), thiosulfate (Daeffler et al., 2017), nitrate (Woo et al., 2020), and aspartate (Landry et al., 2018).

The AtoSC TCS is sensitive to acetoacetate and within E. coli plays a role in short-chain fatty acid (SCFA) metabolism, motility and poly-(R)-3-hydroxybutyrate synthesis (Oshima et al., 2002; Pauli \& Overath, 1972; Pilalis et al., 2011; Theodorou et al., 2013). As shown in Figure 1, acetoacetate is detected by the membrane-bound AtoS histidine kinase (AtoS HK). This then goes on to phosphorylate the AtoC response regulator (AtoC RR). The phosphorylated AtoC RR then induces transcription from the Pato promoter, which subsequently expresses the atoDAEB operon.

Acetoacetate is an extremely important metabolite in mammalian metabolism and energy regulation. It is one of three ketone bodies, alongside -hydroxybutyrate and the less abundant acetone, which can serve as an alternative energy source in the body during periods of low glucose availability (Puchalska \& Crawford, 2017). Typically ketone bodies are found at submillimolar concentrations within the blood; however, these levels can become elevated during periods of extended starvation or intense exercise (Laffel, 1999). Ketone bodies have been linked to protective effects on the neural system, which has led to the use of ketogenic diets as a method for preventing epileptic seizures (Neal et al., 2008). Another study highlighted the role ketone bodies play in stem cell differentiation and homeostasis within the intestines, through the action of -hydroxybutyrate (Cheng et al., 2019). Human mesenchymal stem cells have been shown to have a preference for acetoacetate as an energy-yielding substrate; leading to suggestions that acetoacetate could be added to mesenchymal stem cell culture medium (Board et al., 2017). Acetoacetate has also been shown to act as a signalling molecule for muscle regeneration and can help restore muscle function in a mouse model of muscular wasting (Zou et al., 2016). However, sustained periods of increased ketone body concentrations within humans can be a sign of several pathological states. These include salicylate poisoning, alcoholic ketoacidosis and diabetic ketoacidosis (Laffel, 1999). During extreme cases of diabetic ketoacidosis ketone body concentrations can reach $20 \mathrm{mM}$ and above. If left untreated, this may lead to complications such as a cerebral edema (KanikarlaMarie \& Jain, 2016; Puchalska \& Crawford, 2017). As such, ketone body levels (particularly acetoacetate and -hydroxybutyrate) are monitored regularly in patients with diabetes. Alongside their role in diabetes, ketone bodies are also measured in the blood of cows. During pregnancy, cows become susceptible to ketosis, a condition that adversely affects the health and milk production of dairy cattle (Enjalbert et al., 2001).

It is clear therefore that methods of measuring ketone body levels may find use in a range of biomedical or agricultural applications. Currently, strip tests that use a sodium-nitroprusside reaction, or ketone meters, can be used to monitor ketone levels in urine and blood (Misra \& Oliver, 2015). Although these offer cheap monitoring systems for patients, they are only semiquantitative, and there are a

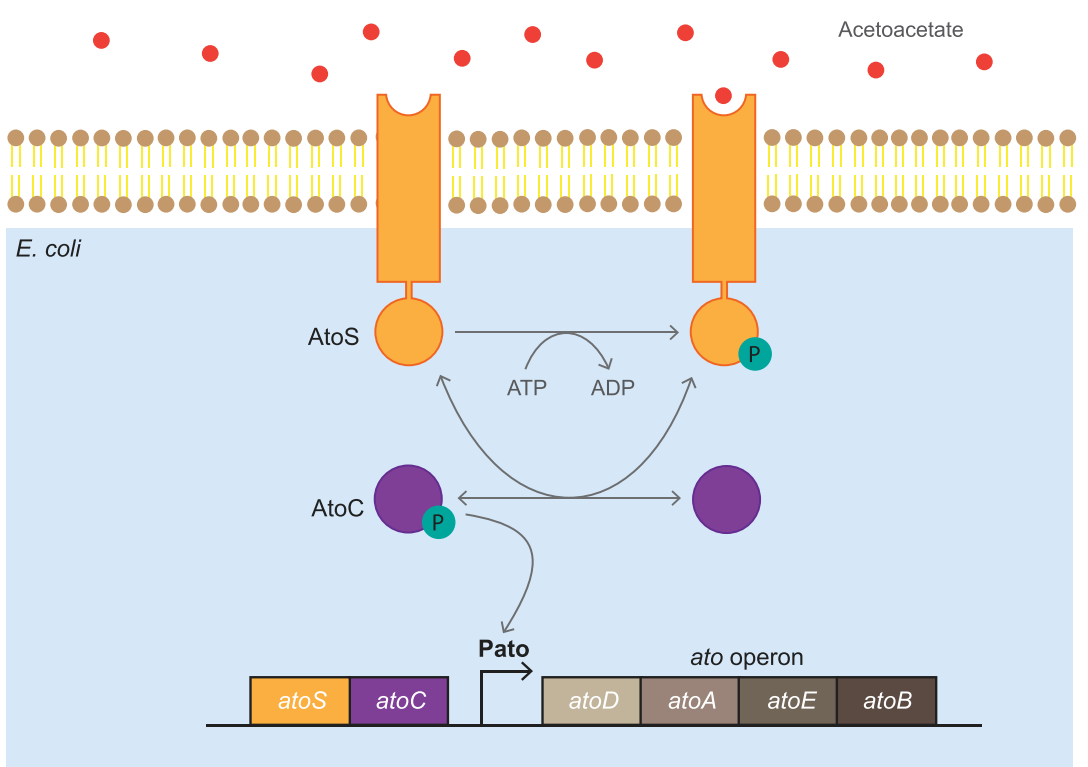

FIGURE 1 Layout of the AtoSC twocomponent system and atoDAEB operon. The AtoS histidine kinase, within the Escherichia coli inner membrane, autophosphorylates in the presence of acetoacetate. The phosphate group is then transferred to the AtoC response regulator, which triggers expression from the Pato promoter 
number of biomedical, scientific, and industrial applications where they are unsuitable. For example, monitoring the production of acetoacetate by engineered E. coli (Hu et al., 2010), exploring how the human microbiota utilises ketone bodies (Sasaki et al., 2020) and investigating host-microbiota interactions in model systems in vivo (Rutter et al., 2019), could all use whole-cell biosensors to report on acetotacetate concentration.

However, biosensors often display observed biosensor behaviour that is mismatched with the desired application, as such there is demand for design strategies that can be used to modify biosensor behaviour without relying on time-consuming trial and error approaches (Gonzalez-Flo et al., 2020). For transcription-factor based biosensors numerous principles have been identified that govern biosensor response, such as modulation of receptor protein expression, via approaches including design of experiments and phenomenological modelling (Berepiki et al., 2020; Chen et al., 2018; Mannan et al., 2017). Further studies have attempted to rationally design more complex TCS-based biosensors. One example used a mathematical model to show that tuning phosphatase activity was able to modify the $K_{1} 2$ of TCS biosensors, where the HK displays both phosphatase and kinase activity (Landry et al., 2018). A further study systematically varied expression of components from a CcaSR TCS biosensor (through varying RBS strength), allowing the authors to optimise biosensor behaviour based on the plasmid library created (Sasaki et al., 2014). Within this study we apply sensitivity analysis to guide the rational design of a TCS biosensor.

Here, we report the construction of E. coli whole-cell biosensors that can be used to detect and report on the presence of acetoacetate. The whole-cell biosensors presented here are based on the AtoSC TCS, found within E. coli. We develop an ordinary differential equation (ODE) model that attempts to capture the action of the AtoSC TCS and use sensitivity analysis of this model to guide the design of an array of acetoacetate-inducible biosensors. The final biosensors display a diverse range of output responses and may be employed in the future for a variety of sensing applications.

\section{2 | MATERIALS AND METHODS}

\subsection{AtoSC plasmid construction}

The plasmids within this study were constructed using the CIDAR MoClo assembly method (Iverson et al., 2016). The oligonucleotides and plasmids designed in this study are given in Tables S1 and S2, respectively, alongside details of their construction.

\subsection{AtoSC biosensor host strains}

A range of host strains were used as chassis for the AtoSC biosensor plasmids. The BW25113 (atoSC ${ }^{+}$), JW2213 (atoS ${ }^{-}$), and JW2214 $\left(a \mathrm{to}^{-}\right)$strains were purchased from the Keio collection (Dharmacon Horizon). The BW28878 (atoSC ${ }^{-}$) double-knockout strain was provided by Professor Kyriakidis (University of Thessaloniki) and E. coli Nissle 1917 by Professor Henderson (University of Birmingham). Competent NEB5 cells were purchased commercially (New England Biolabs). All strains are given in Table S2.

\subsection{AtoSC ODE modelling and sensitivity analysis}

An ODE model, detailed in the Supplementary Information Material, was constructed to describe the AtoSC whole-cell biosensors behaviour (Figure 2a). Simulation and sensitivity analysis of the model was performed in Python using several libraries: NumPy (Harris et al., 2020), SciPy (Virtanen et al., 2020), pandas (McKinney, 2010), SALib (Herman \& Usher, 2017), multiprocessing, joblib, tqdm. Briefly, the system of ODEs was simulated, across a range of AtoS phosphorylation rates $\left(k_{a p}\right)$ as a proxy for acetoacetate concentration, for an arbitrarily long period of time which ensured the system reached equilibrium. Green fluorescent protein (GFP) concentrations at the final time-point produced a dose-response curve to which we fitted a Hill function with basal expression, Equation (1).

$$
\text { GFP }=f^{\min }+\left(f^{\max }-f^{\min }\right) k_{a p}^{n}\left(K^{n}+k_{a p}^{n}\right) .
$$

Parameter sampling followed the method detailed in Campolongo et al. (2007). Specifically, we generated 1000 trajectories and sampled 50 optimal trajectories from this set. Analysis of the sensitivities to each of the Hill function variables, and additionally fold change ( $f^{\max } f^{\min }$ ), to each of the model parameters was performed using the SALib implementation of the Morris method (Campolongo et al., 2007). Plotting was performed in R (Core Team, 2021) using ggplot2 (Wickham, 2016), ggrepel (Slowikowski, 2021), and dplyr (Wickham et al., 2021). The code to reproduce and plot results can be found in a Zenodo repository: doi 10.5281/zenodo.5078479. The equations and parameter bounds used for this model are given in Supporitng Information Section 2.

\section{4 | Growth curve assays}

Overnight bacterial cultures were diluted to an approximate optical density $700\left(\mathrm{OD}_{700}\right)$ of 0.05 , within fresh media. A volume of $120 \mu \mathrm{l}$ of each culture was then added to the well of a 96-well clear bottom microplate (Greiner Bio-One) with a magnetic removable lid. The cultures were incubated in a Tecan Spark plate reader for $2 \mathrm{~h}$ at $37^{\circ} \mathrm{C}$ with shaking at $150 \mathrm{rpm}$ ( $2 \mathrm{~mm}$ amplitude, double orbital) with an $\mathrm{OD}_{700}$ measurement taken every $30 \mathrm{~min}$. Cultures were then induced with the relevant inducer concentration and sealed with a breathe-easy permeable membrane (Diversified Biotech). The plates were then returned to the plate reader and left to grow for a further $16 \mathrm{~h}$, with $\mathrm{OD}_{700}$ measurements taken every $20 \mathrm{~min}$ (the same temperature and shaking conditions were maintained). Inducers were added to the 96-well plates with the aid of an I.DOT liquid handler (Dispendix). Inducers were diluted to the desired stock concentrations and then custom protocols set up within the I.DOT, 
(a)

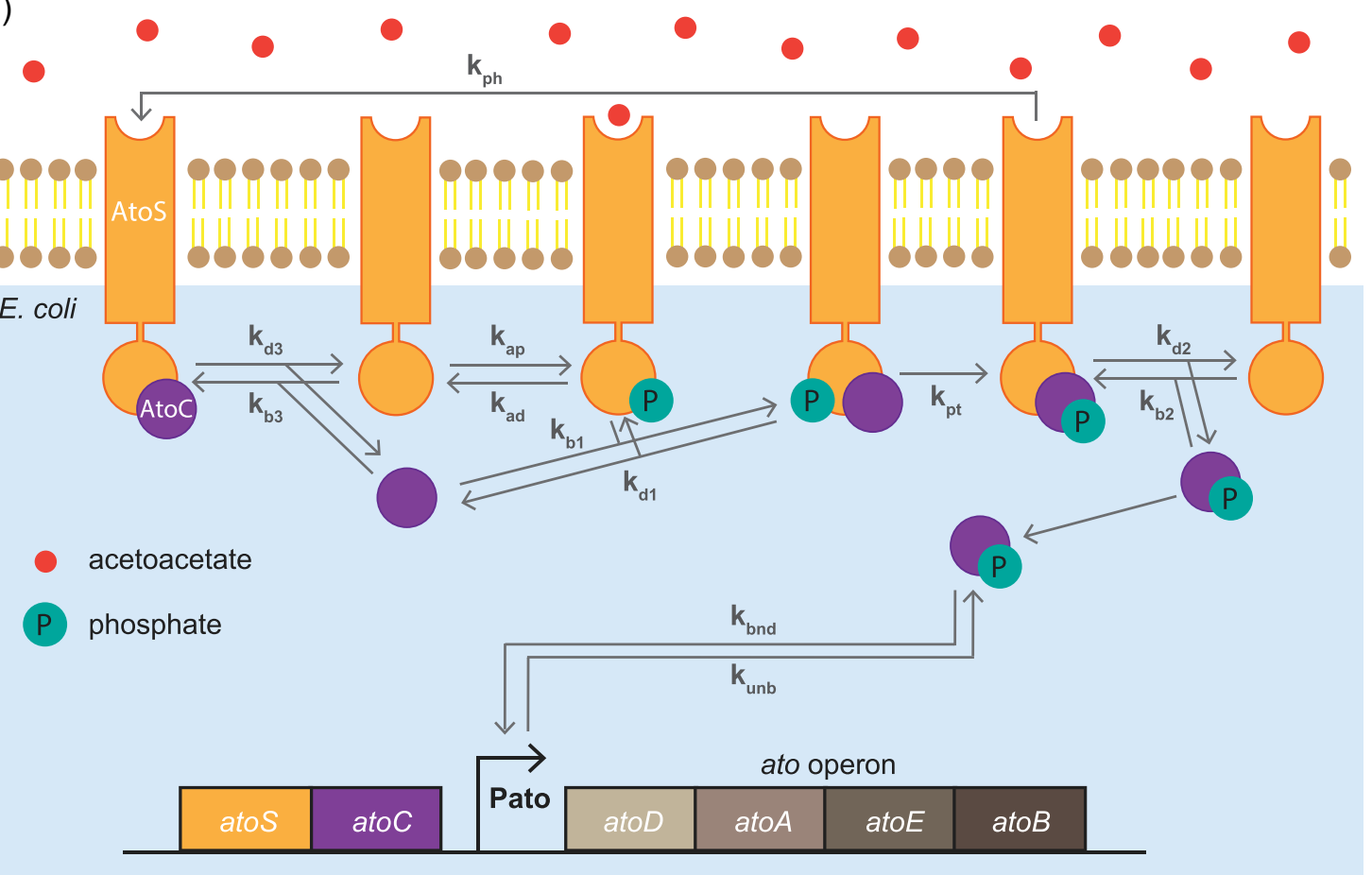

(b)

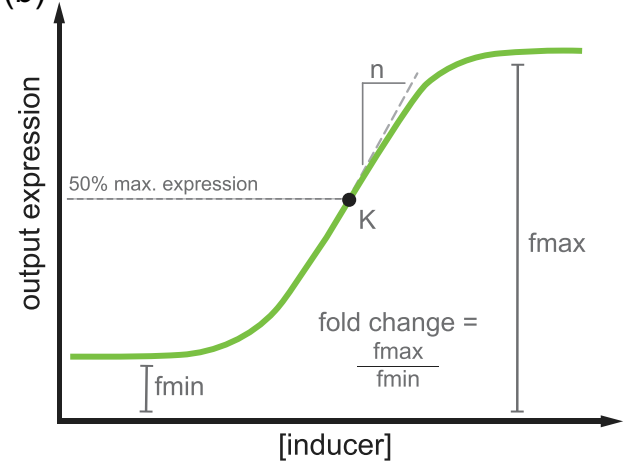

(c)

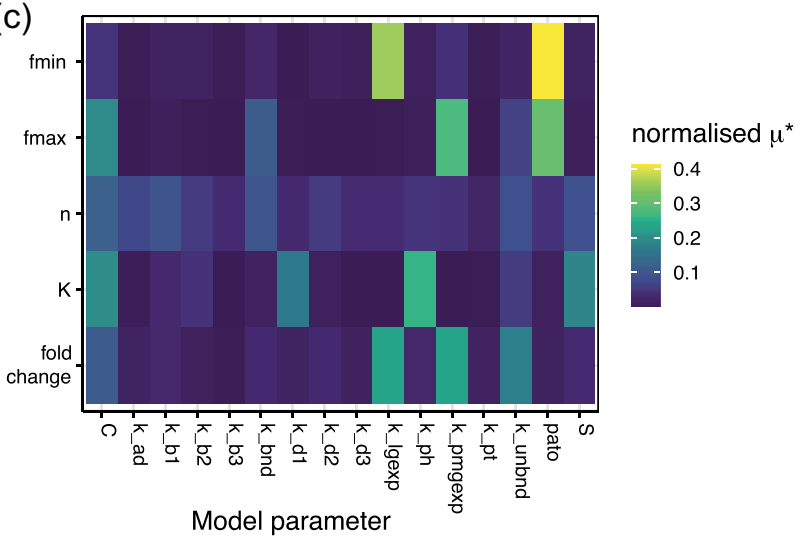

FIGURE 2 Results of the sensitivity analysis conducted on the AtoSC model. (a) Layout of the AtoSC model, illustrating the parameters varied during sensitivity analysis of the system. (b) Illustration of the different aspects of biosensor performance for which sensitivity analysis was performed, ymin and ymax represent $f^{\min }$ and $f^{\max }$, respectively. $K$ represents the $K_{1} 2$ of the biosensor. (c) Heatmap denoting the normalised * results of the sensitivity analysis for the AtoSC model parameters, a higher * value indicates higher parameter influence on biosensor behaviour. "C" refers to AtoC RR concentration, "k_ad" to HK autodephosphorylation, "k_b1" to binding rate of the phosphorylated HK and RR, "k_b2" to the binding of HK to the phosphorylated RR, "k_b3" to the binding of the HK and RR, "k_bnd" to the binding of phosphorylated RR to the Pato promoter, "k_d1" to unbinding of phosphorylated HK and RR, "k_d2" to unbinding of HK and phosphorylated RR, "k_d3" to unbinding of HK and RR, "k_lgexp" to the rate of transcription from uniduced Pato, "k_ph" to dephosphorylation of phosphorylated RR by HK, "k_pmgexp" to the rate of transcription from induced Pato, "k_pt" to the phosphorylation of RR by phosphorylated HK, "k_unbnd" to the unbinding of phosphorylated RR to Pato, "pato" to Pato promoter availability and "S" to AtoS HK concentration (as given in Table S3)

using the I.DOT assay studio software, to automate addition of the correct inducer volumes. Taking the total volume in each well to $125 \mu \mathrm{l}$.

\section{5 | Dose-response assays}

Bacterial cultures, grown overnight in LB media, were diluted to an $\mathrm{OD}_{700}$ of approximately 0.05 in fresh media, and $190 \mu \mathrm{l}$ added to each well of a polypropylene 96-well deep-well plate (Brand, Sigma Aldrich). The plate was then sealed with an autoclaved system Duetz lid and incubated for $2 \mathrm{~h}$ at $37^{\circ} \mathrm{C}$, with $350 \mathrm{rpm}$ shaking (Duetz et al., 2000). Inducers were then added to the desired concentration, bringing the total volume in each well to $200 \mu$ l, the plate was once again sealed with an autoclaved system Duetz lid and the induced cultures incubated at $37{ }^{\circ} \mathrm{C}$, with $350 \mathrm{rpm}$ shaking for $16 \mathrm{~h}$. Samples were taken as required for flow cytometry analysis. 


\section{6 | Flow cytometry analysis}

Flow cytometry was performed on an Attune NxT Acoustic Focusing Cytometer, with an Attune NxT Autosampler (Thermo Fisher Scientific), closely following a previously reported method (Rutter et al., 2019). In brief $1 \mu \mathrm{l}$, of the appropriate sample was diluted 1:200 in sterile phosphate-buffered saline (PBS), in a shallow polystyrene U-bottom 96-well plate. An Attune NxT Autosampler was used to record 10,000 events (for each sample) with three wash and mixing cycles between samples. GFP was excited using a blue laser (488 nm) and detected using a 530/30 nm bandpass filter. Additionally, a sample of 1:300 dilution of rainbow calibration particles in sterile PBS (Spherotech) was recorded allowing for the conversion of arbitrary units to molecules of equivalent fluorophore (MEF), using R commands from the FlopR package (Fedorec et al., 2020).

All collected flow cytometry standard (FCS) data were processed using commands from the FlopR package (Fedorec et al., 2020) and plotted using custom R scripts. Visualisation and curve fitting were performed in R, using the "ggplot2" package and "nls" fitting function (Wickham, 2016). GFP induction data was fit using a Hill function, as given in Equation (1), with $k_{a p}$ representing acetoacetate inducer concentration, $f^{\min }$ minimum fluorescence and $f^{\max }$ maximum recorded fluorescence. Dynamic range was calculated using the following expression, based on the fitted values of $f^{\min }$ and $f^{\max }$ :

$$
\text { Dynamic range }=\frac{f^{\max }-f^{\min }}{f^{\min }} \text {. }
$$

Fold change was calculated as $f^{\max } f^{\min }$

\section{3 | RESULTS}

\subsection{Sensitivity analysis of the AtoSC TCS}

An ODE model describing the action of the AtoSC TCS was developed (Figure 2a) to aid in experimental design. Morris sensitivity analysis was carried out to identify the model parameters that were predicted to have a large effect on AtoSC biosensor response. This analysis was performed for each of the major components of biosensor behaviour, $K_{1} 2, f^{\min }, f^{\max }$, and so on (Figure $2 b$, rankings are given in Figure S1). The sensitivity analysis predicted several parameters would have an influence on final biosensor behaviour. The concentrations of the AtoS HK, AtoC RR proteins and the availability of the Pato promoter (i.e., copy number) were all predicted to have an influence on a range of different aspects of biosensor performance (Figure 2c). In theory, these parameters are readily tuneable experimentally, through varying of plasmid copy number and strength of constitutive expression; this would require no need for the modification of protein sequence/structure or binding affinities. In addition, a successful tuning strategy based on this approach may be portable to other TCS biosensors in future studies. Therefore, we set out to investigate whether varying the concentrations of AtoS HK,
AtoC RR, and Pato promoter would result in changes in final biosensor response, that agree with the predictions returned by sensitivity analysis of the AtoSC TCS model. Further work could explore the modification of other parameters identified as important for determining biosensor behaviour using sensitivity analysis (Figure 2c).

\subsection{Design and construction of an acetoacetate- inducible biosensor}

As described above, the AtoSC TCS consists of the AtoS HK, AtoC RR and Pato promoter. Initially, a basic AtoSC biosensor was constructed by incorporating GFP expression under the control of the Pato promoter on a high-copy pUC based plasmid, designated ASAHO (Figure 3a). The ASAHO plasmid relies on host genomic expression of both AtoS and AtoC. The ASAHO plasmid was initially characterised in three atoSC ${ }^{+}$E. coli strains. As can be seen from Figure 3b,c ASAHO showed a robust increase in GFP expression with increasing acetoacetate concentration in all three strains. The fitted Hill parameters for each of these strains are given in Table S5. The response was similar in all three hosts, with NEB5 ASAHO displaying higher fitted $f^{\max }$ and $K_{1}{ }_{2}$ values. Furthermore, the ASAHO plasmid showed a $K_{1} 2$ commensurate with the concentrations of ketone bodies expected in human blood (submillimolar concentrations), indicating that they are responsive to acetoacetate at physiologically relevant levels (Puchalska \& Crawford, 2017). However, ASAHO was unresponsive to acetoacetate in atoSC- (BW28878), atoS ${ }^{-}$(JW2213), and ato ${ }^{-}$ (JW2214) strains (Figure S3). This indicates that all components of the AtoSC TCS are required for the detection of acetoacetate, and that no other TCSs activate expression from the Pato promoter under these conditions. Growth curves collected for the BW25113 ASAHO WCB showed no substantial change in growth when exposed to increasing concentrations of acetoacetate (Figure S2).

\subsection{Specificity of the acetoacetate-inducible biosensor}

After demonstrating that the AtoSC TCS could be used to construct acetoacetate-inducible biosensors (Figure $3 \mathrm{~b}$ ), alternative inducers were examined to check the specificity of the ASAHO circuit. Six alternative inducers were tested, including the SCFAs acetate, propionate and butyrate, the other ketone bodies, acetone and hydroxybutyrate, and spermidine (which has previously been reported to act as an inducer for the AtoSC TCS) (Theodorou et al., 2007). All were tested at a concentration of $20 \mathrm{mM}$, a concentration above that shown to give maximum acetoacetate induction. When characterised in LB media, acetoacetate produced the highest increase in GFP expression (Figure $3 d$ ), while the alternative inducers showed similar levels of GFP to the uninduced control. As $20 \mathrm{mM}$ is a relatively high concentration, growth assays were measured to ensure the lack of GFP was not caused by toxicity of any of the inducers. All inducers produced no substantial changes in growth 
(a)

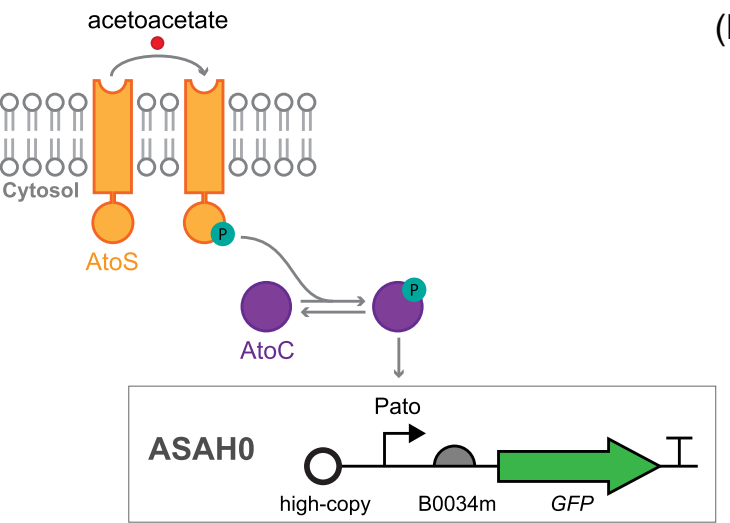

(b)

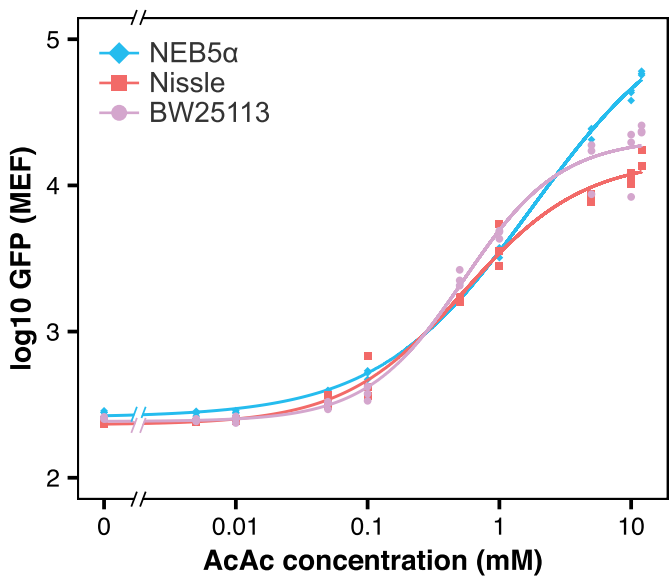

(c)
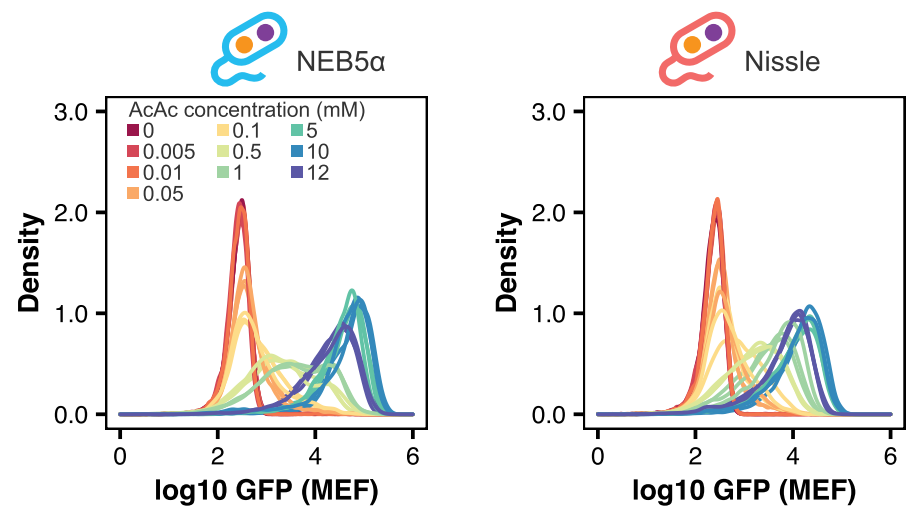

BW25113

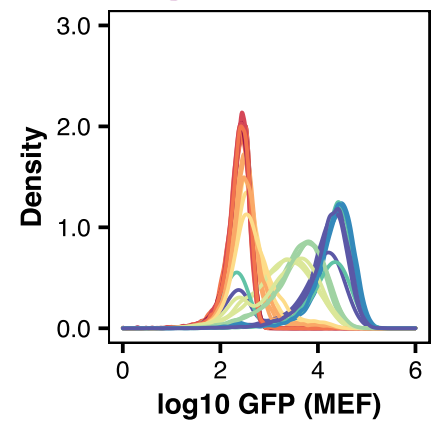

(d)

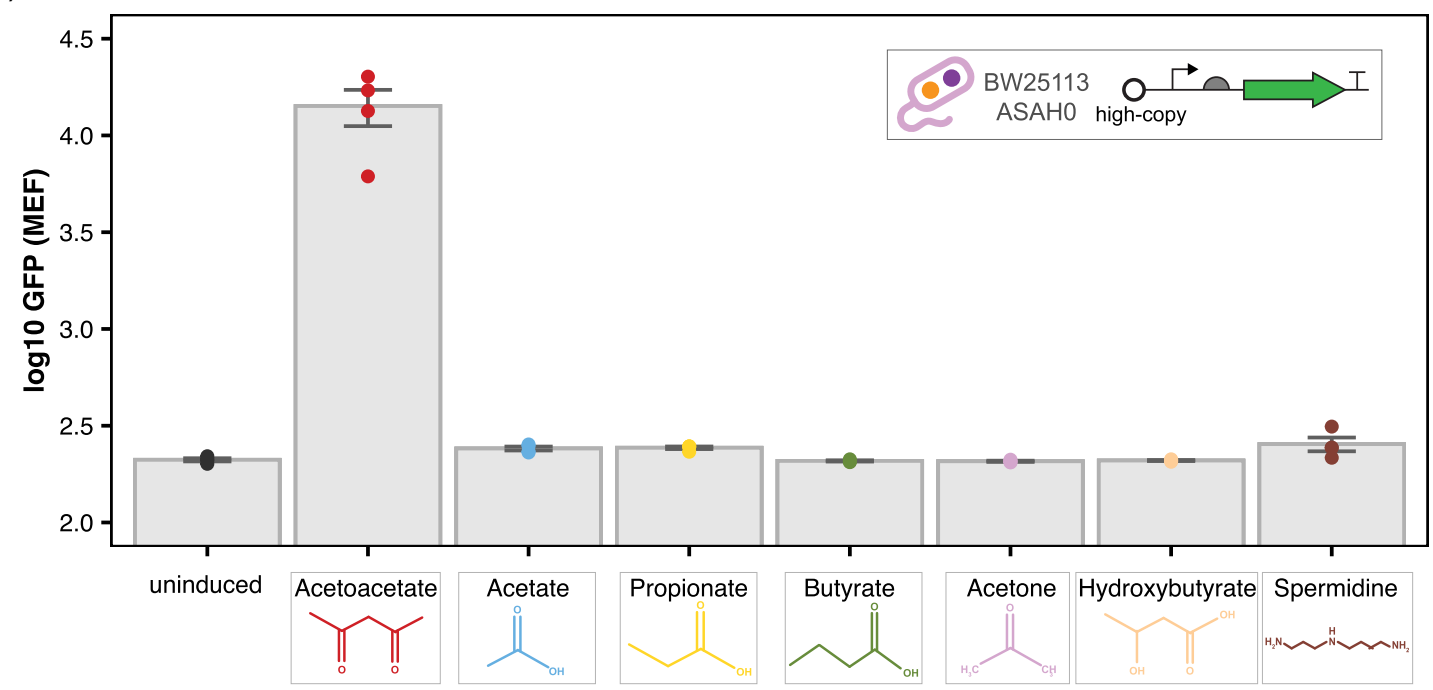

Inducer

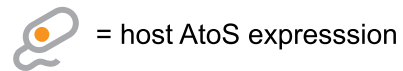

= host AtoC expresssion

FIGURE 3 (See caption on next page) 
FIGURE 4 A comparison of the acetoacetate biosensor response between genomic and plasmid expression of the AtoS and AtoC genes. (a) Layouts of the ASAHO and ASAH2J06 plasmids. ASAH2J06 contains constitutive expression of the AtoS HK and AtoC RR proteins. (b) Median GFP fluorescence of the whole-cell biosensors exposed to acetoacetate (BW25113 ASAHO and BW28878 ASAH2J06: $n=3$ biological repeats, data fit with Hill function, dashed lines indicate the fitted $K_{1 / 2}$ values, BW28878 ASAHO: $n=2$ biological repeats, line indicates lowest measured GFP median, cell symbols indicate the ato $S$ and $a t o C$ presence status in the host chassis genome for each of the three whole-cell biosensor strains). $\mathrm{HK}$, histidine kinase; RR, response regulator (a)

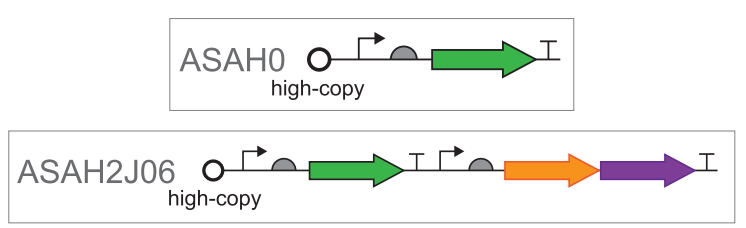

(b)

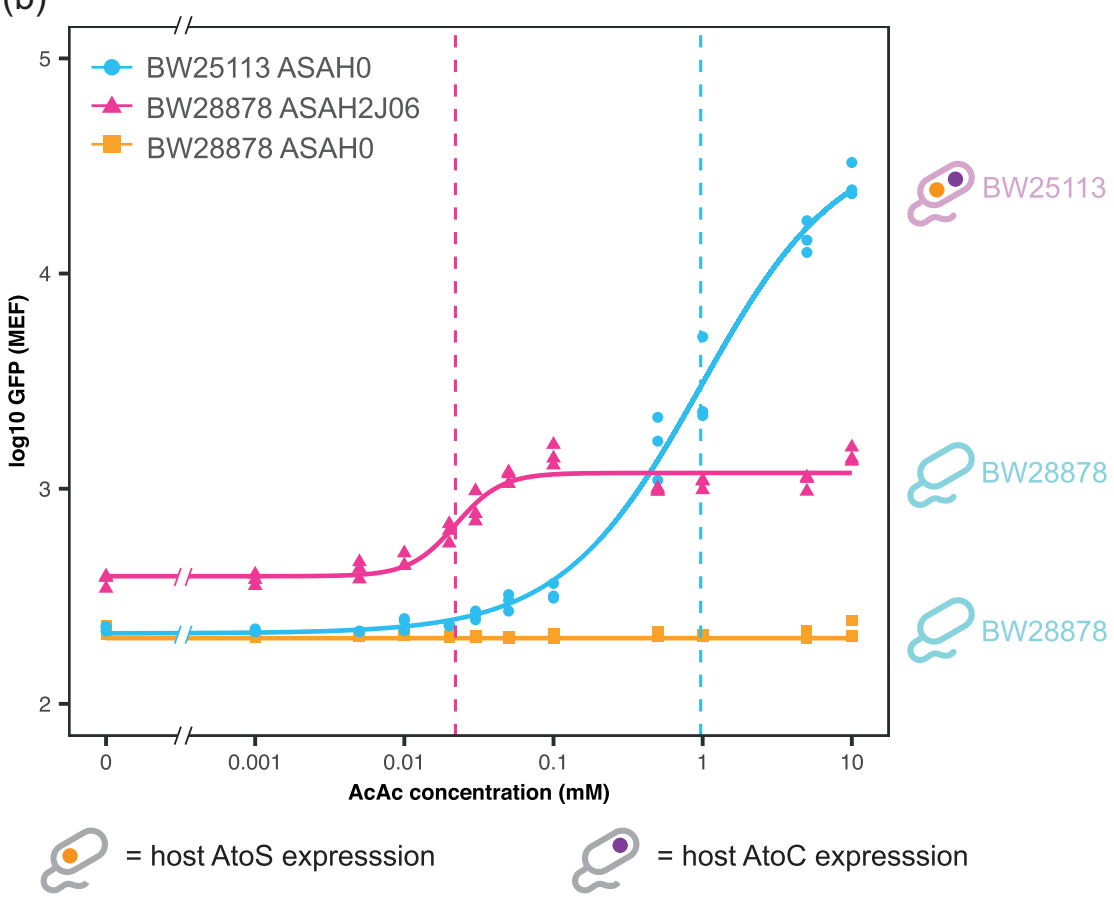

compared to the uninduced control, except for spermidine (Figure S4), which was seen to prevent cell growth. To further examine spermidine induction of BW25113 ASAHO, a full response curve was collected across a range of inducer concentrations (Figure S5). However, no increase in GFP was seen across this range of spermidine concentrations.

Characterisation was also performed for the BW25113 ASAHO biosensor in several different media types (Figures S6 and S7). First, the switching of BW25113 ASAHO in minimal media with glycerol and glucose carbon sources was compared to that in LB media (Figure S6). The biosensor functioned across all three media types, although the fold change was greatly reduced with glucose as the carbon source. In addition, to determine whether the ASAHO biosensor was capable of operating in more complex conditions, the biosensor was characterised in media supplemented with a range of mammalian culture media and HeLa/CHO-K1 conditioned samples (Figure S7). BW25113 ASAHO was found to show induction when exposed to acetoacetate in cultures supplemented with $20 \%$ of these complex samples.

\section{4 | Plasmid versus genome expression of AtoS/ AtoC components}

The ASAHO plasmid relies on host expression of both the AtoS HK and AtoC RR proteins. However, motivated by the results of the sensitivity analysis, we wished to create versions of the AtoSC biosensor where the concentrations of both proteins could be varied (through changes in plasmid copy number). ASAH2J06 was generated through addition of the atoS atoC genes to the ASAHO plasmid, under the control of a constitutive promoter (J23106 of the Anderson promoter library: parts.igem.org); in a manner analogous to a previously reported nitrate biosensor that incorporated the narX narL genes (Woo et al., 2020). All other plasmid components

FIGURE 3 Characterisation of the acetoacetate-sensitive ASAHO plasmid. (a) ASAHO contains GFP under the control of the Pato promoter

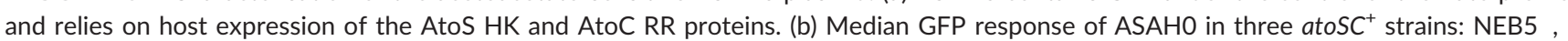
BW25113 and Nissle 1917, exposed to acetoacetate. (c) Density plots of GFP fluorescence for ASAHO in the three atoSC ${ }^{+}$host strains $(n=3$ biological repeats, data fit with Hill function). (d) GFP response of BW25113 ASAHO to a range of alternative inducers in LB media, all at 20 mM ( $n=4$ biological repeats, points show medians and bars mean of medians SE, strain symbols denote host expression of atoS atoC). HK, histidine kinase; MEF, molecules of equivalent fluorophore; RR, response regulator 
were kept the same. The layout of this biosensor is given in Figure 4a. In contrast to $\mathrm{ASAHO}$, the $\mathrm{ASAH} 2 \mathrm{JO} 6$ biosensor produced an acetoacetate-inducible change in GFP expression even when host expression of both the ato $S$ and the ato $C$ genes were knocked-out (Figure S8). To explore the effect of plasmid versus host gene expression in more detail the responses of ASAHO in BW25113 (only host expression of AtoS/AtoC) and ASAH2J06 in BW28878 (only plasmid expression of AtoS/AtoC) were directly compared (Figure 4b). BW25113 ASAHO displayed a greater $f^{\text {max }}$, dynamic range and lower $f^{\text {min }}$ than ASAH2J06. However, BW28878 ASAH2J06 showed a fitted $K_{1} 2$ value an order of magnitude lower than the BW25113 ASAHO biosensor $(31.7 \pm 3.78$ vs. $344 \pm 42.1 \mu \mathrm{M}$, respectively). The source of AtoS HK and AtoC RR expression were the only differences between these strains. However, these relatively simple changes produced a dramatic shift in the response curves of the BW25113 ASAHO and BW28878 ASAH2J06 biosensors, supporting the predictions gained through sensitivity analysis of the AtoSC model.

\subsection{Engineering modified biosensor responses by varying AtoS, AtoC, and Pato concentrations}

As the BW25113 ASAHO and BW28878 ASAH2J06 biosensors displayed such different responses to acetoacetate induction we wished to explore if further changes to the concentrations of AtoS, AtoC, and the Pato promoter would result in other behaviours. To this end, a range of further AtoSC plasmids were produced. Low copy versions of both the ASAHO and ASAH2J06 plasmids were created by replacing the origin of replications with a low copy SC101 origin. These low copy plasmids were designated ASALO and ASAL2J06, respectively. Furthermore, the atoC genes were removed from the ASAH2J06 and ASAL2J06 plasmids to produce the ASAH1J06 and ASAL1J06 plasmids, which have plasmid expression of the AtoS HK but rely on host expression of the AtoC RR. The response curves for all six of these biosensors are given in Figure 5.

Increasing the copy number of the plasmid containing only the Pato promoter produced a large increase in the $f^{\max }$ of the final GFP response, while having no noticeable effect on $f^{\text {min }}$ (Figure $5 b$ ). Increasing the copy number of the plasmid containing both Pato and the AtoS HK (ASAH1J06/ASAL1J06) reversed this trend, with the lower copy number displaying a higher $f^{\max }$. Again little difference was seen in $f^{\min }$ (Figure $5 \mathrm{~d}$ ). Finally, increasing the copy number of the plasmid containing Pato, the AtoS HK and AtoC RR (ASAH2J06) ASAL2J06) resulted in a vertical shift of the GFP response, increasing both $f^{\min }$ and $f^{\max }$ (Figure 5f). All of the fitted parameters to these response curves are given in Table S5. Of all the ato knockout (BW25113, BW28878, JW2213, and JW2214) whole-cell biosensor strains constructed, BW25113 ASAHO displayed the largest dynamic range (114), whereas BW28878 ASAH2J06 was found to have the lowest $K_{1} 2(31.7 \pm 3.78 \mu \mathrm{M})$ followed by the low-copy BW28878 ASAL2J06 $(40.1 \pm 2.28 \mu \mathrm{M})$. From these response curves it is clear that introducing variations in the concentrations of AtoS $\mathrm{HK}$, AtoC $\mathrm{RR}$, and Pato promoter, through varying plasmid versus host genome expression and plasmid copy number, can produce dramatically (a)

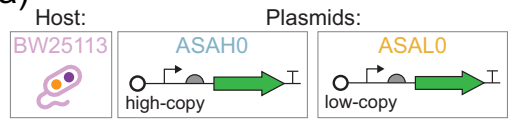

(b)

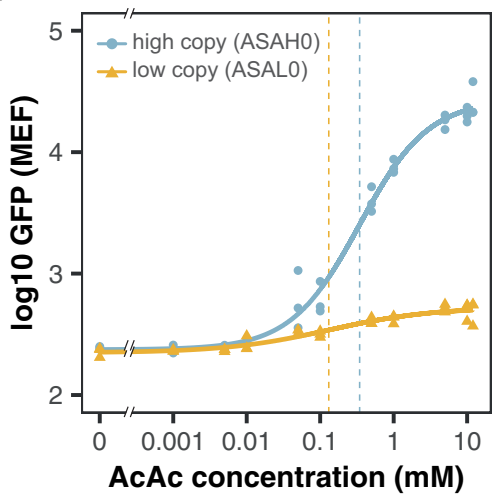

(c)

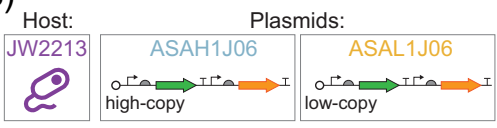

(d)

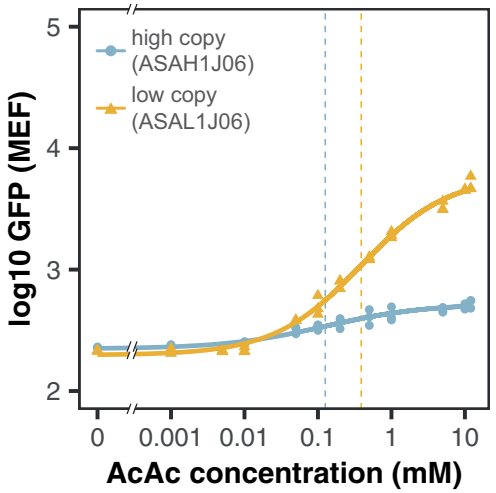

(e)

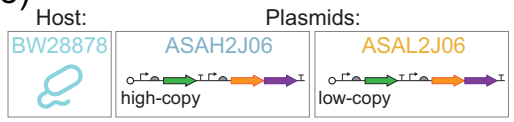

(f)

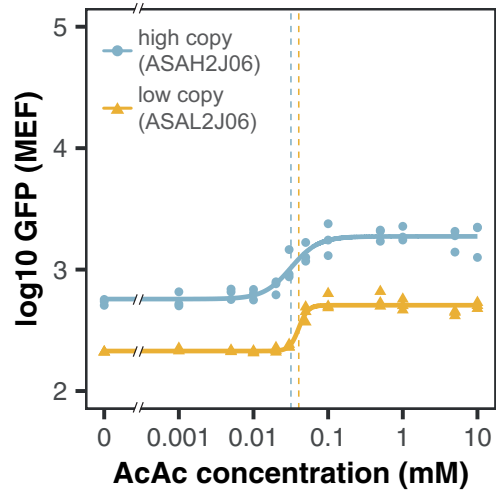

$=$ host AtoS expresssion

$=$ host AtoC expresssion

FIGURE 5 Manipulating whole-cell biosensor response by changing the copy number of components of the AtoSC two-component system. (a) Plasmid layouts of ASAHO and ASALO. (b) Median GFP response of ASAHO and ASALO in the atoSC ${ }^{+}$BW25113 host strain. (c) Plasmid layouts of ASAH1J06 and ASAL1J06. (d) Median GFP response of ASAH1J06 and ASAL1J06 in the atoS ${ }^{-}$JW2213 host strain. (e) Plasmid layouts of ASAH2J06 and ASAH2J06. (f) Median GFP response of ASAH2J06 and ASAL2J06 in the atoSC- BW28878 host strain ( $n=3$ biological repeats, data fit with Hill function, dashed lines indicate fitted $K_{1} 2$ values) 
different response curves and associated behaviours, as qualitatively predicted by sensitivity analysis of the AtoSC model.

\section{4 | DISCUSSION}

\section{1 | Insights from sensitivity analysis}

The AtoSC TCS is a complex system with a large number of different species and parameters involved in its functioning. This presents a challenging task when trying to decide which parameters to modify during circuit engineering. Morris sensitivity analysis was performed to identify the parameters that might have the greatest influence on biosensor behaviour, and therefore the largest scope for effective engineering of the final biosensor response. When ranking the Morris sensitivity analysis all parameters involving the reporter elements (e.g., GFP maturation/degradation) were removed from the results since biosensors can be built with a range of reporters and outputs (Lopreside et al., 2019). We wished to focus on the parameters influencing the TCS, as these features may have wider value for engineering other TCS-based biosensors. From the final rankings, it can be seen that the concentration of AtoC RR ("C"), the concentration of AtoS HK ("S"), and Pato promoter concentration ("pato"), were predicted to have an effect on a range of different aspects of biosensor behaviour (Figure 2c). These results provided motivation for the whole-cell biosensor designs characterised within Figures 4 and 5 , as changing the availability of these three main components was hypothesised to have a large effect on biosensor behaviour. It should be noted that other parameters were also predicted to have an effect on biosensor behaviour (e.g., the binding rate of the phosphorylated AtoC RR to the Pato promoter). It may be possible to use these so far unexplored parameters as a starting point for further attempts to engineer acetoacetate biosensors with different responses in the future. In addition, further work could include the fitting of TCS parameters from time-course data, thereby improving the predictive capability of the model.

\subsection{Using the AtoSC TCS to create acetoacetate- inducible biosensors}

The ASAHO plasmid is the simplest design for an acetoacetate sensitive biosensor that can be created from the AtoSC TCS, requiring host expression of the AtoS HK and AtoC RR. As shown in Figure S2, increasing exposure to acetoacetate did not appear to produce substantial changes in the exponential growth rate of BW25113 ASAHO. E. coli is naturally able to metabolise acetoacetate as a carbon source using genes contained within the ato operon. However, the growth curves collected here suggest that this metabolism is negligible, in LB media, as no increase in growth rate was seen. ASAHO was initially tested in three E. coli strains: NEB5 (a commercial cloning strain), BW25113 (the parent strain of the Keio knockout collection; Baba et al., 2006) and Nissle 1917 (a commonly used microbiome engineering strain; Ou et al., 2016). Although the ASAHO design is simple and was found to be acetoacetate-inducible in all three strains, it provides fewer components that can be modified to tune biosensor performance. The aim of the ASAH2J06 design was to provide a self-contained plasmid that would (a) not rely on host expression of AtoS HK/AtoC RR, and (b) provide a greater potential design space from which to produce the desired biosensor behaviour. Upon construction both ASAHO and ASAH2J06 were found to be acetoacetate-inducible (Figure 4).

As mentioned previously, the typical concentrations of ketone bodies within a healthy adult are submillimolar. It has been reported that these may increase to approximately $1.0 \mathrm{mM}$ during hyperketonemia, above $3.0 \mathrm{mM}$ during ketoacidosis and reach levels in excess of $20 \mathrm{mM}$ during cases of uncontrolled diabetes (Kanikarla-Marie \& Jain, 2016; Laffel, 1999). The original form of the whole-cell biosensor, BW25113 ASAHO, was able to sense and respond to acetoacetate concentrations within this physiologically relevant range, and displayed a $K_{1} 2$ lower than the detection limit of the commercially available Ketostix, which rely on a sodium nitroprusside reaction to detect levels of acetoacetate in a semiquantitative manner ( $K_{1} 2$ of $344 \pm 42.1 \mu \mathrm{M}$ vs. detection limit of $\sim 500 \mu \mathrm{M}$, respectively). These are desirable characteristics if the biosensor is to be used for diagnostic applications. However, it should be noted that the relative levels of the different ketone bodies may change across different diseased states. For example, in a healthy adult the ratio of hydroxybutyrate to acetoacetate commonly lies between 1:1 and 3:1, however, during cases of acute diabetic ketoacidosis this ratio can vary greatly due to impaired interconversion of -hydroxybutyrate to acetoacetate (Kanikarla-Marie \& Jain, 2016). Therefore, the actual proportion of acetoactetate may vary depending on the disease state. This means that more sensitive biosensors (i.e., biosensors with a lower $K_{1}{ }_{1}$ ) may be required for certain diagnostic applications. As can be seen from Figure 4, the modified BW28878 ASAH2J06 biosensor showed a substantially lower $K_{1} 2$ than the original BW25113 ASAHO (31.7 \pm 3.78 vs. $344 \pm 42.1 \mu \mathrm{M}$, respectively). As expected, BW28878 ASAHO showed no response to acetoacetate induction (Figures 4 and S3), as the AtoS HK and AtoC RR are not expressed (either from the genome or plasmid) within this strain. These results suggest that replacing host genome with plasmid expression could offer a starting point for attempts to reduce the $K_{1} 2$ of other TCS-based whole-cell biosensors.

The BW25113 ASAHO biosensor was found to be specific to acetoacetate when tested against a range of alternative inducers. As the AtoSC TCS and the genes of the atoDAEB operon are known to play a role in SCFA metabolism the SCFAs acetate, propionate, and butyrate were tested as alternative inducers. The data collected here supports previous results that have reported SCFAs, particularly butyrate, can not induce the AtoSC TCS (Pauli \& Overath, 1972; Theodorou et al., 2011). In addition, the two other ketone bodies, acetone and -hydroxybutyrate were tested for induction; neither were found to induce the BW25113 ASAHO biosensor. As discussed previously, spermidine has been reported elsewhere as an alternative inducer of the AtoSC TCS (Theodorou et al., 2007). Characterisation 
of BW25113 ASAHO did not show an increase in GFP response when exposed to spermidine (Figures $3 d$ and S5). However, it is possible that the spermidine concentrations needed to trigger GFP production from this plasmid may be above those that become toxic to the cells. Future work could explore whether spermidine induction of the BW25113 ASAHO biosensor can be seen in other culture conditions. To further explore the switching response of BW25113 ASAHO, the biosensor was characterised in a range of more complex media components (Figures S6 and S7). BW25113 ASAHO displayed an increase in GFP upon exposure to acetoacetate in all the conditions tested; however, the fold change of the switching was found to be much lower when grown in M9 media with glucose as the carbon source (Figure S6). Previous work has shown that the ato $D$ gene (present at the beginning of the atoDAEB operon) is a target of the Hfq-binding small RNA, Spot 42, which suppresses numerous metabolic genes in the presence of a preferred carbon source (Beisel et al., 2012). This is a likely mechanism for the repression seen with the BW25113 ASAHO strain. Future work could explore methods of reducing this catabolic repression within the engineered whole-cell biosensor strains.

A range of AtoSC biosensor strains, with differing expression of AtoS HK, AtoC RR, and Pato promoter were also characterised (Figure 5). From the response curves it is clear that varying these parameters was able to produce a variety of different biosensor behaviours. This supports the results of the sensitivity analysis, which identified the concentrations of these parameters as important for governing behaviour of the biosensors. However, it should be noted that the agreement between in silico and in vivo results is qualitative and cannot be used to quantitatively predict the behaviour of the final biosensor design. Our knowledge of the regulatory interactions influencing the AtoSC TCS is incomplete and therefore our model does not capture the full biological complexity of this system. In future, the model reported here will be adapted as our knowledge of AtoSC TCS biology improves, in turn helping to improve the predictive capability of our sensitivity analysis approach.

\section{5 | CONCLUSIONS}

Our study has demonstrated that the AtoSC TCS can be used to construct acetoacetate-inducible biosensors. Characterisation of the BW25113 ASAHO whole-cell biosensor showed that the biosensor is specific to acetoacetate, with no GFP response recorded when exposed to a range of alternative inducer molecules. Sensitivity analysis performed on a model of the AtoSC TCS predicted that varying AtoS $\mathrm{HK}, \mathrm{AtoC} \mathrm{RR}$, and Pato promoter presence would have a large effect on the final biosensor response. Subsequently, we constructed a range of AtoSC whole-cell biosensors with varying expression of AtoS $\mathrm{HK}$, AtoC RR, and presence of the Pato promoter. These whole-cel biosensors were found to exhibit different response curves when exposed to acetoacetate, supporting the insights gained through sensitivity analysis. In the future, these AtoSC whole-cell biosensors may be incorporated into a range of other biomedical, bioproduction, or agricultural applications, where there is a need to monitor the levels of the acetoacetate ketone body. The design methods used here may also be applied to the construction of other TCS biosensors, where there is a mismatch between the desired and observed biosensor behaviour.

\section{ACKNOWLEDGEMENTS}

J.W.R received a UKRI-BBSRC PhD studentship through the LIDo DTP. G.M.T. was supported by funding from the BBSRC (grant no. BB/S003681/1). C.P.B. acknowledges funding from the European Research Council (ERC) under the European Unions Horizon 2020 research and innovation programme (grant no. 770835). C.P.B and J.W.R acknowledge funding from the Wellcome Trust (grant no. 209409/Z/17/Z)

\section{CONFLICT OF INTERESTS}

The authors declare that there are no conflict of interests.

\section{DATA AVAILABILITY STATEMENT}

The experimental data used to create the plots in this manuscript and the code used for modelling is freely available at: 10.5281/zenodo. 5078479.

\section{REFERENCES}

Baba, T., Ara, T., Hasegawa, M., Takai, Y., Okumura, Y., Baba, M., Datsenko, K. A., Tomita, M., Wanner, B. L., \& Mori, H. (2006). Construction of Escherichia coli K12 inframe, singlegene knockout mutants: The Keio collection. Molecular Systems Biology, 2(1). 20060008. https://doi.org/10.1038/msb4100050

Beisel, C. L., Updegrove, T. B., Janson, B. J., \& Storz, G. (2012). Multiple factors dictate target selection by $\mathrm{Hfq}$-binding small RNAs. The EMBO Journal, 31(8), 1961-1974. https://doi.org/10.1038/emboj. 2012.52

Berepiki, A., Kent, R., Machado, L. F. M., \& Dixon, N. (2020). Development of high-performance whole cell biosensors aided by statistical modeling. ACS Synthetic Biology, 9(3), 576-589. https://doi.org/10. 1021/acssynbio.9b00448

Board, M., Lopez, C., van den Bos, C., Callaghan, R., Clarke, K., \& Carr, C. (2017). Acetoacetate is a more efficient energy-yielding substrate for human mesenchymal stem cells than glucose and generates fewer reactive oxygen species. The International Journal of Biochemistry \& Cell Biology, 88, 75-83. https://doi.org/10.1016/j. biocel.2017.05.007

Campolongo, F., Cariboni, J., \& Saltelli, A. (2007). An effective screening design for sensitivity analysis of large models. Environmental Modelling \& Software, 22(10), 1509-1518. https://doi.org/10. 1016/j.envsoft.2006.10.004

Chen, X., Xia, X., Lee, S. Y., \& Qian, Z. (2018). Engineering tunable biosensors for monitoring putrescine in Escherichia coli. Biotechnology and Bioengineering, 115(4), 1014-1027. https://doi.org/10.1002/bit.26521

Cheng, C.-W., Biton, M., Haber, A. L., Gunduz, N., Eng, G., Gaynor, L. T., Tripathi, S., Calibasi-Kocal, G., Rickelt, S., Butty, V. L., MorenoSerrano, M., Iqbal, A. M., Bauer-Rowe, K. E., Imada, S., Ulutas, M. S., Mylonas, C., Whary, M. T., Levine, S. S., Basbinar, Y., ...Yilmaz, Ö.H. (2019). Ketone body signaling mediates intestinal stem cell homeostasis and adaptation to diet. Cell, 178(5), 1115-1131. https://doi.org/10.1016/j.cell.2019.07.048

Courbet, A., Endy, D., Renard, E., Molina, F., \& Bonnet, J. (2015) Detection of pathological biomarkers in human clinical samples via 
amplifying genetic switches and logic gates. Science Translational Medicine, 7(289), 289ra83. https://doi.org/10.1126/scitranslmed. aaa3601

Daeffler, K. N., Galley, J. D., Sheth, R. U., OrtizVelez, L. C., Bibb, C. O., Shroyer, N. F., Britton, R. A., and Tabor, J. J. (2017). Engineering bacterial thiosulfate and tetrathionate sensors for detecting gut inflammation. Molecular Systems Biology, 13(4), 923. https://doi.org/ 10.15252/msb.20167416

Duanmu, D., Bachy, C., Sudek, S., Wong, C.-H., Jimenez, V., Rockwell, N. C., Martin, S. S., Ngan, C. Y., Reistetter, E. N., van Baren, M. J., Price, D. C., Wei, C.-L., Reyes-Prieto, A., Lagarias, J. C., and Worden, A. Z. (2014). Marine algae and land plants share conserved phytochrome signaling systems. Proceedings of the National Academy of Sciences, 111(44), 15827-15832. https:// doi.org/10.1073/pnas.1416751111

Duetz, W. A., Ruedi, L., Hermann, R., OConnor, K., Buchs, J., \& Witholt, B. (2000). Methods for intense aeration, growth, storage and replication of bacterial strains in microtiter plates. Applied and Environmental Microbiology, 66(6), 2641-2646.

Enjalbert, F., Nicot, M., Bayourthe, C., \& Moncoulon, R. (2001). Ketone bodies in milk and blood of dairy cows: Relationship between concentrations and utilization for detection of subclinical ketosis. Journal of Dairy Science, 84(3), 583-589. https://doi.org/10.3168/ jds.S0022-0302(01)74511-0

Fedorec, A. J. H., Robinson, C. M., Wen, K. Y., \& Barnes, C. P. (2020). FlopR: An open source software package for calibration and normalization of plate reader and flow cytometry data. ACS Synthetic Biology, 9(9), 2258-2266. https://doi.org/10.1021/acssynbio.0c00296

Goers, L., Ainsworth, C., Goey, C. H., Kontoravdi, C., Freemont, P. S., \& Polizzi, K. M. (2017). Whole-cell Escherichia coli lactate biosensor for monitoring mammalian cell cultures during biopharmaceutical production. Biotechnology and Bioengineering, 114(6), 1290-1300. https://doi.org/10.1002/bit.26254

Gonzalez-Flo, E., Alaball, M. E., \& Macia, J. (2020). Two-component biosensors: Unveiling the mechanisms of predictable tunability. ACS Synthetic Biology, 9(6), 1328-1335. https://doi.org/10.1021/ acssynbio.0c00010

Harris, C. R., Millman, K. J., van der Walt, S. J., Gommers, R., Virtanen, P., Cournapeau, D., Oliphant, T. E., Wieser, E., Taylor, J., Berg, S., Smith, N. J., Kern, R., Picus, M., Hoyer, S., van Kerkwijk, M. H., Brett, M., Haldane, A., del Río, J. F., Wiebe, M., ...Oliphant, T. E. (2020). Array programming with NumPy. Nature, 585(7825), 357-362. https://doi.org/10.1038/s41586-020-2649-2

Herman, J., \& Usher, W. (2017). SALib: An open-source python library for sensitivity analysis. The Journal of Open Source Software, 2(9). 97. https://doi.org/10.21105/joss.00097

Hicks, M., Bachmann, T. T., \& Wang, B. (2020). Synthetic biology enables programmable cell-based biosensors. ChemPhysChem, 21(2), 132-144. https://doi.org/10.1002/cphc.201900739

Hoch, J. A. (2000). Two-component and phosphorelay signal transduction. Current Opinion in Microbiology, 3(2), 165-170. https://doi.org/10. 1016/S1369-5274(00)00070-9

Hu, D., Shi, Z., Wu, Q., \& Chen, G.-Q. (2010). Microbial production of acetoacetate by recombinant Escherichia coli. Bioresource Technology, 101(21), 8477-8480. https://doi.org/10.1016/j.biortech.2010.06.021

Iverson, S. V., Haddock, T. L., Beal, J., \& Densmore, D. M. (2016). CIDAR MoClo: Improved MoClo assembly standard and new E. coli part library enable rapid combinatorial design for synthetic and traditional biology. ACS Synthetic Biology, 5(1), 99-103. https://doi. org/10.1021/acssynbio.5b00124

Jia, X., Zhao, T., Liu, Y., Bu, R., \& Wu, K. (2018). Gene circuit engineering to improve the performance of a whole-cell lead biosensor. FEMS Microbiology Letters, 365(16). https://doi.org/10.1093/ femsle/fny157
Jung, K., Fabiani, F., Hoyer, E., \& Lassak, J. (2018). Bacterial transmembrane signalling systems and their engineering for biosensing. Open Biology, 8(4), 180023. https://doi.org/10.1098/ rsob.180023

Kanikarla-Marie, P., \& Jain, S. K. (2016). Hyperketonemia and ketosis increase the risk of complications in type 1 diabetes. Free Radical Biology and Medicine, 95, 268-277. https://doi.org/10.1016/j. freeradbiomed.2016.03.020

Laffel, L. (2018). Ketone bodies: A review of physiology, pathophysiology and application of monitoring to diabetes. Diabetes/Metabolism Research and Reviews, 15(6), 412-426. https://doi.org/10.1002/ (SICI)1520-7560(199911/12)15:6\%3C412::AID-DMRR72\%3E3.0. $\mathrm{CO} ; 2-8$

Landry, B. P., Palanki, R., Dyulgyarov, N., Hartsough, L. A., \& Tabor, J. J. (2018). Phosphatase activity tunes two-component system sensor detection threshold. Nature Communications, 9(1), 1433. https://doi. org/10.1038/s41467-018-03929-y

Lin, C., Zhang, Q.-X., \& Yeh, Y.-C. (2019). Development of a whole-cell biosensor for the determination of tyrosine in urine for point-of-care diagnostics. Analytical Methods, 11(10), 1400-1404. https://doi.org/ 10.1039/C9AY00070D

Lopreside, A., Wan, X., Michelini, E., Roda, A., \& Wang, B. (2019). Comprehensive profiling of diverse genetic reporters with application to whole-cell and cell-free biosensors. Analytical Chemistry, 91(23), 15284-15292. https://doi.org/10.1021/acs. analchem.9b04444

Mahbub, K. R., Krishnan, K., Naidu, R., \& Megharaj, M. (2017). Development of a whole cell biosensor for the detection of inorganic mercury. Environmental Technology \& Innovation, 8, 64-70. https://doi.org/10.1016/j.eti.2017.04.003

Mannan, A. A., Liu, D., Zhang, F., \& Oyarzún, D. A. (2017). Fundamental design principles for transcription-factor-based metabolite biosensors. ACS Synthetic Biology, 6(10), 1851-1859. https://doi. org/10.1021/acssynbio.7b00172

Mascher, T., Helmann, J. D., \& Unden, G. (2006). Stimulus perception in bacterial signal-transducing histidine kinases. Microbiology and Molecular Biology Reviews, 70(4), 910-938. https://doi.org/10. 1128/MMBR.00020-06

McKinney, W. (2010). Data structures for statistical computing in Python. In S. van der Walt and J. Millman, eds., Proceedings of the 9th Python in Science Conference (pp. 56-61). 10.25080/Majora-92bf1922-00a.

Misra, S., \& Oliver, N. S. (2015). Utility of ketone measurement in the prevention, diagnosis and management of diabetic ketoacidosis. Diabetic Medicine, 32(1), 14-23. https://doi.org/10.1111/dme. 12604

Neal, E. G., Chaffe, H., Schwartz, R. H., Lawson, M. S., Edwards, N., Fitzsimmons, G., Whitney, A., \& Cross, J. H. (2008). The ketogenic diet for the treatment of childhood epilepsy: A randomised controlled trial. The Lancet Neurology, 7(6), 500-506. https://doi. org/10.1016/S1474-4422(08)70092-9

OConnor, J. R., Kuwada, N. J., Huangyutitham, V., Wiggins, P. A., \& Harwood, C. S. (2012). Surface sensing and lateral subcellular localization of WspA, the receptor in a chemosensory-like system leading to c-di-GMP production. Molecular Microbiology, 86(3), 720-729. https://doi.org/10.1111/mmi.12013

Oshima, T., Aiba, H., Masuda, Y., Kanaya, S., Sugiura, M., Wanner, B. L., Mori, H., and Mizuno, T. (2002). Transcriptome analysis of all twocomponent regulatory system mutants of Escherichia coli K-12. Molecular Microbiology, 46(1), 281-291. https://doi.org/10.1046/j. 1365-2958.2002.03170.x

Ou, B., Yang, Y., Tham, W. L., Chen, L., Guo, J., \& Zhu, G. (2016). Genetic engineering of probiotic Escherichia coli Nissle 1917 for clinical application. Applied Microbiology and Biotechnology, 100(20), 8693-8699. https://doi.org/10.1007/s00253-016-7829-5 
Papenfort, K., \& Bassler, B. L. (2016). Quorum sensing signalresponse systems in Gram-negative bacteria. Nature Reviews Microbiology, 14(9), 576-588. https://doi.org/10.1038/nrmicro.2016.89

Pauli, G., \& Overath, P. (1972). Pato operon: A highly inducible system for acetoacetate and butyrate degradation in Escherichia coli. European Journal of Biochemistry, 29(3), 553-562. https://doi.org/10.1111/j. 1432-1033.1972.tb02021.x

Pilalis, E., Chatziioannou, A. A., Grigoroudis, A. I., Panagiotidis, C. A., Kolisis, F. N., \& Kyriakidis, D. A. (2011). Escherichia coli genomewide promoter analysis: Identification of additional AtoC binding target elements. BMC Genomics, 12(1), 238. https://doi.org/10. 1186/1471-2164-12-238

Puchalska, P., \& Crawford, P. A. (2017). Multi-dimensional roles of ketone bodies in fuel metabolism, signaling, and therapeutics. Cell Metabolism, 25(2), 262-284. https://doi.org/10.1016/j.cmet.2016.12.022

R Core Team. (2021). R: A language and environment for statistical computing. R Foundation for Statistical Computing [Computer software manual]. Vienna, Austria. https://www.R-project.org/

Riglar, D. T., Giessen, T. W., Baym, M., Kerns, S. J., Niederhuber, M. J., Bronson, R. T., Kotula, J. W., Gerber, G. K., Way, J. C., and Silver, P. A. (2017). Engineered bacteria can function in the mammalian gut long-term as live diagnostics of inflammation. Nature Biotechnology, 35(7), 653-658. https://doi.org/10.1038/ nbt.3879

Rutter, J. W., Ozdemir, T., Galimov, E. R., Quintaneiro, L. M., Rosa, L. Thomas, G. M., Cabreiro, F., and Barnes, C. P. (2019). Detecting changes in the caenorhabditis elegans intestinal environment using an engineered bacterial biosensor. ACS synthetic biology, 8(12), 2620-2628. https://doi.org/10.1038/emboj.2012.52

Sasaki, K., Sasaki, D., Hannya, A., Tsubota, J., \& Kondo, A. (2020). In vitro human colonic microbiota utilises d- -hydroxybutyrate to increase

butyrogenesis. Scientific reports, 10(1), 1-8.

Sasaki, K., Sasaki, D., Hannya, A., Tsubota, J., \& Kondo, A. (2014). Refactoring and optimization of light-switchable Escherichia coli two-component systems. ACS Synthetic Biology, 3(11), 820-831. https://doi.org/10.1021/sb500273n

Singh, K. K. (2000). The Saccharomyces cerevisiae sln1p-ssk1p twocomponent system mediates response to oxidative stress and in an oxidant-specific fashion. Free Radical Biology and Medicine, 29(10), 1043-1050. https://doi.org/10.1016/S0891-5849(00)00432-9

Slowikowski, K. (2021). ggrepel: Automatically position non-overlapping text labels with 'ggplot2' [Computer software manual]. R package version 0.9.1. https://CRAN.R-project.org/package=ggrepel

Stock, A. M., Robinson, V. L., \& Goudreau, P. N. (2000). Two-component signal transduction. Annual Review of Biochemistry, 69(1), 183-215. https://doi.org/10.1146/annurev.biochem.69.1.183

Stocker, J., Balluch, D., Gsell, M., Harms, H., Feliciano, J., Daunert, S., Malik, K. A., and van der Meer, J. R. (2003). Development of a set of simple bacterial biosensors for quantitative and rapid measurements of arsenite and arsenate in potable water. Environmental Science \& Technology, 37(20), 4743-4750. https://doi.org/10.1021/es034258b

Theodorou, E. C., Theodorou, M. C., \& Kyriakidis, D. A. (2013). Regulation of poly-(R)-(3-hydroxybutyrate-co-3-hydroxyvalerate) biosynthesis by the AtoSCDAEB regulon in phaCAB Escherichia coli. Applied Microbiology and Biotechnology, 97(12), 5259-5274. https://doi.org/ 10.1007/s00253-013-4843-8
Theodorou, E. C., Theodorou, M. C., Samali, M. N., \& Kyriakidis, D. A. (2011). Activation of the AtoSC two-component system in the absence of the AtoC $\mathrm{N}$-terminal receiver domain in $\mathrm{E}$. coli. Amino Acids, 40(2), 421-430. https://doi.org/10.1007/s00726010-0652-x

Theodorou, M. C., Theodorou, E. C., Panagiotidis, C. A., \& Kyriakidis, D. A. (2007). Spermidine triggering effect to the signal transduction through the AtoS-AtoC/Az two-component system in Escherichia coli. Biochimica et Biophysica Acta (BBA)-General Subjects, 1770(8), 1104-1114. https://doi.org/10.1016/j.bbagen.2007.03.009

Virtanen, P., Gommers, R., Oliphant, T. E., Haberland, M., Reddy, T., Cournapeau, D., Burovski, E., Peterson, P., Weckesser, W., Bright, J., van der Walt, S. J., Brett, M., Wilson, J., Millman, K. J., Mayorov, N., Nelson, A. R. J., Jones, E., Kern, R., Larson, E., ...SciPy 1.0 Contributors. (2020). SciPy 1.0: Fundamental algorithms for scientific computing in Python. Nature Methods, 17, 261-272. https://doi.org/ 10.1038/s41592-019-0686-2

Wang, B., Hicks, M., \& Bachmann, T. T. (2019). Synthetic biology enables programmable cell-based biosensors. ChemPhysChem, 21, 132-144. https://doi.org/10.1002/cphc.201900739

Wen, K. Y., Rutter, J. W., Barnes, C. P., \& Dekker, L. (2020). Fundamental building blocks of whole-cell biosensor design. In Handbook of cell biosensors, (pp. 1-23). Springer International Publishing.

Wickham, H. (2016). ggplot2: Elegant graphics for data analysis. SpringerVerlag.

Wickham, H., Franois, R., Henry, L., \& Mller, K. (2021). dplyr: A grammar of data manipulation [Computer software manual]. $\mathrm{R}$ package version 1.0.6. https://CRAN.R-project.org/package $=$ dplyr

Woo, S.-G., Moon, S.-J., Kim, S. K., Kim, T. H., Lim, H. S., Yeon, G.-H., Sung, B. H., Lee, C.-H., Lee, S.-G., Hwang, J. H., and Lee, D.-H. (2020). A designed whole-cell biosensor for live diagnosis of gut inflammation through nitrate sensing. Biosensors and Bioelectronics, 168, 112523. https://doi.org/10.1016/j.bios. 2020.112523

Zou, X., Meng, J., Li, L., Han, W., Li, C., Zhong, R., Miao, X., Cai, J., Zhang, Y., and Zhu, D. (2016). Acetoacetate accelerates muscle regeneration and ameliorates muscular dystrophy in mice. Journal of Biological Chemistry, 291(5), 2181-2195. https://doi. org/10.1074/jbc.M115.676510

\section{SUPPORTING INFORMATION}

Additional Supporting Information may be found online in the supporting information tab for this article.

How to cite this article: Rutter, J. W., Dekker, L., Fedorec, A. J. H., Gonzales, D. T., Wen, K. Y., Tanner, L. E. S., Donovan, E., Ozdemir, T., Thomas, G. M., \& Barnes, C. P. (2021).

Engineered acetoacetate-inducible whole-cell biosensors based on the AtoSC two-component system. Biotechnology and Bioengineering, 1-12. https://doi.org/10.1002/bit.27897 